\title{
Pour une meilleure lisibilité des factures médicales
}

\section{Urs Stoffel}

Dr méd., membre du Comité central, responsable du département Médecine et tarifs ambulatoires

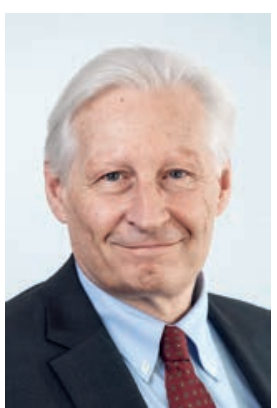

Dans le dernier volet de mesures visant à freiner la hausse des coûts de la santé, le Conseil fédéral exige notamment:

«Les fournisseurs de prestations sont légalement tenus de faire parvenir systématiquement une copie de la facture aux patients. Ceux-ci peuvent ainsi vérifier leurs factures; ils prennent davantage conscience des coûts. En cas de non-respect de cette règle, le fournisseur de prestations peut être sanctionné en vertu de l'art. 59 LAMal.»

Compliquées, opaques voire illisibles pour les non-initiés: les factures médicales sont un thème récurrent dans les médias, que ce soit dans la presse écrite mais aussi à la radio et à la télévision.

Si ce formulaire est aussi complexe, c'est parce qu'il n'a pas été conçu pour le contrôle des factures par les patients.

Si de nombreuses tentatives ont eu lieu pour résoudre ce problème (aides à la lecture, applications chargées de «traduire» les factures), aucune n'a vraiment convaincu jusqu'ici. Car c'est forcément toujours lorsque la facture arrive dans la boîte aux lettres et que l'on souhaite la vérifier que l'on n'a pas ces outils sous la main.

La FMH se félicite de cette mesure proposée par le Conseil fédéral. Nous sommes convaincus que le patient est seul en mesure de vérifier si les prestations facturées ont bel et bien été fournies, et de jouer ainsi un rôle déterminant dans le contrôle des factures. Mais pourquoi ces factures sont-elles aussi compliquées? Lors de l'introduction du TARMED en 2004, différents acteurs de la santé - associations des fournisseurs de prestations et des assureurs - se sont regroupés au sein du Forum Datenaustausch, ${ }^{1}$ chargé d'établir des standards communs pour l'échange électronique de données. Le formulaire XML actuellement en vigueur pour rédiger les factures a été fixé de manière contraignante par le Forum; chaque nouvelle version ou modification doit recevoir l'aval de l'ensemble des membres. Si ce formulaire est aussi complexe, c'est parce qu'il a avant tout été conçu pour que les assureurs puissent traiter les factures électroniquement et les contrôler comme la loi les y oblige. Autrement dit, son but premier est de transmettre l'intégralité des informations nécessaires aux assureurs, et non de permettre aux patients de contrôler leurs factures. Mais ce n'est certainement pas l'intention de la FMH de compliquer la tâche des patients et de les empêcher de contrôler leurs factures. Bien au contraire; la FMH met tout en œuvre pour que les factures soient simples, lisibles et qu'elles puissent être vérifiées facilement par les patients.

Fort de ce constat, le Comité central de la FMH a décidé d'examiner la possibilité d'améliorer la lisibilité de ce formulaire et la compréhension des différentes positions tarifaires. Une solution consisterait à transmettre aux patients un récapitulatif clair des prestations avec le justificatif de remboursement pour les

La FMH continue à mettre tout en œuvre pour que ce problème de lisibilité des factures médicales appartienne prochainement au passé.

assureurs. Dans tous les cas, la FMH continue à s'investir pour que ce problème de lisibilité des factures médicales appartienne prochainement au passé. 